\title{
ON CERTAIN SUPPORT POINTS OF THE CLASS $S$
}

\author{
LOUIS BRICKMAN ${ }^{1}$ AND STEPHAN RUSCHEWEYH
}

\begin{abstract}
A support point of $S$ maps the unit disk onto the complement of an analytic arc going to $\infty$. We study the case where this arc has an analytic continuation through its finite endpoint and back to $\infty$. As an application we find that only under severe restrictions can a Bazilevix function be a support point of $S$.
\end{abstract}

Let $\Delta$ be the open unit disk in the complex plane, and let $H(\Delta)$ be the usual topological linear space of holomorphic functions on $\Delta$. The class $S$ is the subset of $H(\Delta)$ consisting of univalent functions $f$ with the normalization $f(0)=f^{\prime}(0)-1=$ 0 . Let $L$ be an element of the dual space $H(\Delta)^{*}$ of $H(\Delta)$; that is, let $L$ be complexvalued, linear, and continuous on $H(\Delta)$. Then the functional $\operatorname{Re} L$ achieves a maximum on $S$, and if $L$ is nonconstant on $S$, any extremal function $f$ maps $\Delta$ onto the complement of an analytic arc $\Gamma_{f}$ satisfying

$$
L\left(\frac{f^{2}}{f-w}\right)\left(\frac{d w}{w}\right)^{2}>0 \quad\left(w \in \Gamma_{f}\right)
$$

$[6,4,2]$. (This can fail at the endpoint of $\Gamma_{f}$, but only if $f$ is a rotation of the Koebe function.) Also $[4,2]$, there is a line to which $\Gamma_{f}$ is asymptotic at $\infty$. Such an extremal function $f$ is called a support point of $S$.

For the functionals $L$ usually studied-for example coefficient functionals or, more generally, point evaluation of a derivative of some order at a point of $\Delta-$ the expression $L\left(f^{2} /(f-w)\right)$ is a rational function of $w$. In this note we assume this is the case and we study the situation where $\Gamma_{f}$ has an analytic continuation through its finite endpoint and back to $\infty$. (Known examples occur when $\Gamma_{f}$ is a half line with radial angle at the endpoint less than or equal to $\pi / 4$. This is due to K. Pearce [3].) We prove that this analytic extension of $\Gamma_{f}$ must pass through $\infty$ analytically, or in other words must be a closed analytic curve on the Riemann sphere. As a consequence we find that no Bazilevic function-except Pearce's examples - can be a support point of $S$. We have been unable to determine whether the closed analytic curve must be a circle, that is, whether the known examples are the only ones possible. Also unsettled is the question of whether our assumption that $L\left(f^{2} /(f-w)\right)$ is rational can be dropped either in the theorem below or in the corollary concerning Bazilevic functions. Our theorem, then, is the following.

THEOREM. Suppose $f$ is a support point of $S$ with respect to the functional $L \in H(\Delta)^{*}$. Assume that

(a) $L\left(f^{2} /(f-w)\right)$ is a rational function of $w$, and

Received by the editors September 16, 1983.

1980 Mathematics Subject Classification. Primary 30C55, 30C70, 30C45.

${ }^{1}$ This work was done at Würzburg University and supported by the Deutsche Forschungs gemeinschaft. 
(b) $\Gamma_{f}$, the omitted arc of $f$, has an analytic extension $\Gamma$ through the finite tip and going back to $\infty$.

Then, in a neighborhood of $\infty, \Gamma$ is the conformal image of a line segment. In particular there is a (single) line to which $\Gamma$ is asymptotic at both ends.

Proof. Let $\Gamma=\gamma(I)$, where $I$ is a real interval and $\gamma$ is analytic on $I$. We define the function $\varphi$ by

$$
\varphi(t)=L\left[\frac{f^{2}}{f-\gamma(t)}\right]\left[\frac{\gamma^{\prime}(t)}{\gamma(t)}\right]^{2}, \quad t \in I .
$$

Then by (a), $\varphi$ is analytic on $I$ except possibly for poles, and by (1), $\varphi$ is positive on a subinterval of $I$. It follows that $\varphi$ is real valued on $I$ (except possibly for poles). Now, the quadratic differential in (1) has a simple pole at $\infty[\mathbf{7}, \mathbf{4}]$, while the "model" for quadratic differentials with a simple pole is $(1 / \zeta) d \zeta^{2}$ (simple pole at 0 ) $[5$, p. 213]. This means that the quadratic differential (1) can be obtained from the model by a conformal substitution $\zeta=h(w)$ taking $\infty$ to 0 , and therefore that the trajectory structure of (1) near $\infty$ conformally the same as that of the model near 0 . (Note that for any analytic arc $\alpha$ near $\infty$, the value of $L\left(f^{2} /(f-w)\right)(d w / w)^{2}$ at $w=\alpha(t)$ is equal to $(1 / \zeta) d \zeta^{2}$ at $\zeta=h(\alpha(t))$.) But the only analytic arcs going to 0 on which $(1 / \zeta) d \varsigma^{2}$ is real lie on $\mathbf{R}$. Hence, near $\infty, \Gamma$ is the conformal image of an interval $(-\varepsilon, \varepsilon)$ (namely by $\left.h^{-1}\right)$. The final assertion follows easily. We remark that our proof remains valid as long as $L\left(f^{2} /(f-w)\right)$ has only isolated singularities in $\mathbf{C}$.

To apply the iheorem to Bazilevic functions we begin by recalling that $f$ is Bazilevic of type $\gamma=\alpha+i \beta(\alpha>0, \beta \in \mathbf{R})$ if

$$
f(z)=\left[\int_{0}^{z} \gamma g(x)^{\alpha} h(x) x^{i \beta-1} d x\right]^{1 / \gamma} .
$$

Here $g$ is a normalized $\left(g(0)=0, g^{\prime}(0)=1\right)$ starlike function, and $h$ is a subordinate to some function of the form $(1-a z) /(1-z),|a|=1$. The "half line functions" mentioned before the theorem can of course be obtained by choosing $\gamma=1, g(z)=$ $z /(1-b z)^{2}, h(z)=(1-a z) /(1-b z)(|a|=|b|=1, a \neq b)$. We suppose now that such a function $f$ is a support point of $S$. Then $\mathbf{C} \backslash f(\Delta)$ is an analytic arc $\Gamma_{f}$, and it follows from $\left[1\right.$, Theorem 2] that $\Gamma_{f}=l^{1 / \gamma}$, where $l$ is some half line. (We conjecture that another proof of this fact can be obtained by showing that for arbitrary $\gamma,(3)$ defines a single-slit mapping if and only if $g$ and $h$ are chosen exactly as above. Then, for $z=e^{i \theta}$,

$$
\frac{d}{d \theta} f(z)^{\gamma}=i z \frac{d}{d z} f(z)^{\gamma}=i \gamma z^{i \beta}\left[\frac{z}{(1-b z)^{2}}\right]^{\alpha} \frac{1-a z}{1-b z} .
$$

Since $z^{i \beta}$ is positive, $\left[z /(1-b z)^{2}\right]^{\alpha}$ has only one argument, and $(1-a z) /(1-b z)$ has only two possible arguments, it follows that $\left\{f\left(e^{i \theta}\right)^{\gamma}\right\}$ is a half line.) Calculations show that if $\beta \neq 0, l^{1 / \gamma}$ has no asymptotic direction at $\infty$, while if $\beta=0$ and $0<\alpha<1, l^{1 / \alpha}$ has an asymptotic direction but no asymptotic line. Hence $\gamma=\alpha \geq 1$. Next, assuming $f$ is not a Koebe function, we conclude that the line $L$ containing $l$ does not pass through 0 . Therefore $\Gamma_{f}$ has the analytic continuation $L^{1 / \alpha}$, and condition (b) of the theorem is satisfied. But $L^{1 / \alpha}$ satisfies the conclusion of the theorem only if $\alpha=1$. Indeed, another calculation shows that if $\alpha>1, L^{1 / \alpha}$ 
has two asymptotic rays separated by an angle of $\pi / \alpha$. Thus we have proved the following result.

COROLlaRY. Let $f$ be a Bazilevic function and also a support point of $S$ with corresponding function $L\left(f^{2} /(f-w)\right)$ rational. Then $\Gamma_{f}$ is a half line.

We conclude by describing a situation in which condition (b) of the theorem obtains (and here we thank Peter Duren for a helpful discussion). Suppose $f \in S$ and $f$ is a support point with respect to two "essentially different" linear functionals, $L$ and $J$. By this we mean that it is not the case that $L(h)=a h(0)+b h^{\prime}(0)+c J(h)$ for certain constants $a, b, c$ and for all $h \in H(\Delta)$. We suppose also that $\left.L\left(f^{2} / f-w\right)\right)$ and $J\left(f^{2} /(f-w)\right)$ are rational functions of $w$ with quotient $q(w)$. Then, by (1) and the corresponding version of (1) for $J, q$ is nonnegative on $\Gamma_{f}$. Moreover $q$ is not constant. Indeed,

$$
q=c \Rightarrow(L-c J)\left(f^{2} /(f-w)\right) \equiv 0 \Rightarrow(L-c J)(h)=a h(0)+b h^{\prime}(0),
$$

contradicting "essentially different". Now from $q$ being rational and nonconstant it can be shown that $q^{-1}(\mathbf{R} \cup\{\infty\})$ is a finite union of closed analytic arcs on the sphere $\mathbf{C} \cup\{\infty\}$. Since $\Gamma_{f}$ is a subset of this union, (b) follows. The special case where $L=a_{n}$ and $J=a_{m}(m>n \geq 2)$ was discussed by A. K. Bahtin, Ukrainian Math. J., 1981, who showed that $f$ must be a rotation of the Koebe function. It would be interesting to know whether this conclusion holds in the present, more general, context.

\section{REFERENCES}

1. F. G. Avkadiev and L. A. Aksent'ev, Functions of the Bazilevix class in the disc and in an annulus, Dokl. Akad. Nauk SSSR 214 (1974); English transl. in Soviet Math. Dokl. 15 (1974).

2. L. Brickman and D. R. Wilken, Support points of the set of univalent functions, Proc. Amer. Math. Soc. 42 (1974), 523-528.

3. K. Pearce, New support points of $S$ and extreme points of $H S$, Proc. Amer. Math. Soc. 81 (1981), $425-428$.

4. A. Pfluger, Lineare Extremalprobleme bei schlichten Funktionen, Ann. Acad. Sci. Fenn. Ser. AI Math. 489 (1971).

5. Ch. Pommerenke, Univalent functions, Vandenhoeck and Ruprecht, Göttingen, 1975.

6. M. Schiffer, A method of variation within the family of simple functions, Proc. London Math. Soc. 44 (1938), 432-449.

7. 95-101. MR 37 \#4249.

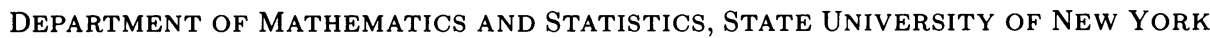
at Albany, Albany, New YORK 12222

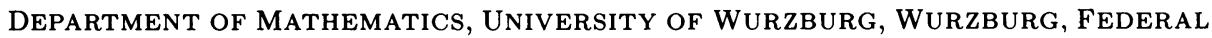
REPUBLIC OF GERMANY 